\title{
Improving solar cell efficiency with optically optimised TCO layers
}

\author{
K. Fleischer ${ }^{\mathrm{a}, *}$, E. Arca ${ }^{\mathrm{a}}$, I.V. Shvets ${ }^{\mathrm{a}}$ \\ ${ }^{a}$ Cleaner Energy Laboratory, School of Physics, Trinity College Dublin, Dublin 2, Ireland
}

\begin{abstract}
In this paper we analyse the capabilities and limitations of single layer index matching coatings for the glass/TCO or TCO/absorber interfaces in thin film solar cells. Optical models are used to evaluate the potential gain in cell efficiencies with hypothetical ideal matching materials to minimise losses due to reflections at internal interfaces. We suggest the range of desired optical properties of such materials, where a net gain in cell efficiency can be achieved, and discuss the commercial benefits of such intermediate matching layer. For some selected ternary and quaternary novel oxides available, the potential efficiency gain due to the inclusion of an anti-reflective matching layer in a solar cell structure is calculated and compared with the case of ideal matching materials.
\end{abstract}

Keywords: photovoltaic, solar cell, coating, anti-reflection, efficiency, optical

\section{Introduction}

The potential of photovoltaic energy for addressing the world's growing energy demand is well known. However solar energy remains an expensive technology and efficiencies of commercial modules have not risen significantly in recent years. One problem is the transfer of highly efficient laboratory cell designs into a commercial production. Hence cost reduction by upscaling the production and more efficient material use has been the driving force in the solar industry in recent years. The fastest growing market segment is low cost, low efficiency thin films cells. These cells offer greater output power for the same costs, usually quantified in $\$ / \mathrm{W}_{p}[1,2]$.

Single absorber thin film cells have rather simple layer structures, usually a front glass substrate, a TCO front contact, the active $p-n$ or $p-i-n$ junction and a metallic back contact. The choice of the active layer in such thin film cells is driven by the production of good quality $\mathrm{p}-\mathrm{n}$ junctions of a material with a band gap optimised for the potential efficiency (ShockleyQueisser limit [3]). Today, cells based on a-Si, $\mu c-S i, \mathrm{CdTe}$, or $\mathrm{CuIn}(\mathrm{Se} / \mathrm{S})$ active layers are used. Indium tin oxide (ITO), aluminated zinc oxide (AZO), $\mathrm{ZnO}: \mathrm{B}$ (BZO) or flourinated tin oxide $\left(\mathrm{SnO}_{2}: \mathrm{F}, \mathrm{FTO}\right)$ are used as transparent front contact material. However only the latter three are suitable for large scale production, as ITO is too expensive [4].

Simple cell designs lead to reflective losses at the air-glass, glass-TCO, and TCO-absorber interface which add to reflective total losses of $15-20 \%$ of incident light at normal incidence. At oblique angles this amount further increases to $20-25 \%$ at $30^{\circ}$ or $30-40 \%$ at $60^{\circ}$ angle of incidence. For the air/glass interface, concepts for reducing these losses do exist and include either a patterning or dielectric coatings of the front glass, mostly composed of $\mathrm{SiO}_{2}$ nanoclusters. For low cost thin film solar

\footnotetext{
${ }^{*}$ Corresponding author

Email address: fleisck@tcd.ie (K. Fleischer)
}

cells however only the latter has any commercial significance. $\mathrm{SiO}_{2}$ nanoclusters are already commercially available and have close to ideal dielectric properties for broad band anti-reflective layers [5]. For this reason we will limit our discussion to the glass/TCO and TCO/absorber interface. For these interfaces, until recently [6], no direct anti-reflective interface layers are used by present day commercial thin film technologies. We will show though, that one technology relevant to our discussion, that has gained a more widespread use - the roughening the TCO surface - can be equivalent to an anti-reflective layer in the low haze regime. Even a small roughness does lead to a reduction in the $\mathrm{TCO} /$ absorber reflective losses in addition to the more often discussed changes in the light path for a large roughness, enhancing the absorption in the active layer [7-10].

In this paper we analyse the effect of single layer dielectric coatings on the reduction of unwanted interface reflections in thin film solar cells. We also analyse if the case for development of novel TCOs with a matched refractive index has economic merits. For this we consider the cost implications of additional layers and compare these with benefits of the increased cell output. Only single layer coatings are considered and only normal incidence efficiency improvements are calculated. Although we will only present normal incidence simulations, the matching layers will, in contrast to texturing, also be effective at lower angles of incidence and the improvements in cell efficiency for oblique illumination will be higher.

\section{Mathematical modelling}

\subsection{Multilayer calculations}

We calculated the reflectance of a multilayer stack, using the transfer matrix formalism $[11,12]$. The stack for the solar cell used in our calculation is shown in Fig. 1. As the effects discussed can be applied to a variety of absorber and TCO materials, we chose to model a relatively simple stack consisting of 


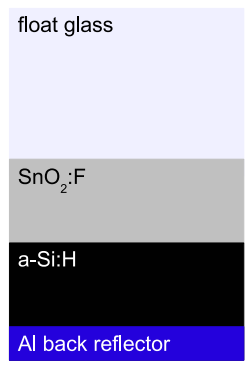

a) b) TCO/absorber (TA)

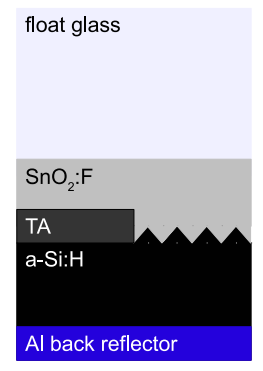

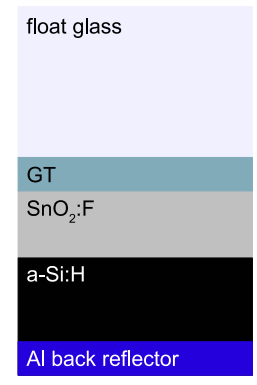

c) glass/TCO (GT)
Figure 1: Schematics of layer stacks used in simulation. a) the reference structure of a thin film solar cell consisting of the float glass substrate, TCO layer $\left(650 \mathrm{~nm}, \mathrm{SnO}_{2}: \mathrm{F}\right)$ and absorber $(300 \mathrm{~nm}$ a-Si:H). b) stack including an interface layer between the TCO and the absorber, consisting of either an additional dielectric layer (marked TA) or a roughened interface leading to a mixed interface layer. c) the stack including a glass/TCO anti-reflective layer (marked GT).

float glass, $650 \mathrm{~nm} \mathrm{SnO}_{2}: \mathrm{F}$ as TCO and $300 \mathrm{~nm}$ amorphous silicon as absorber. All media have been considered homogeneous with abrupt interfaces. In some cases a rough TCO/absorber interface has been modelled, treating the rough area as an effective medium mixture (Bruggeman, fill factor 0.5, [13]). Such an effective medium approximation (EMA) can describe the change in reflectance, caused by the presence of an interface area with a different refractive index, due to the mixture of two different materials. This method is frequently used to describe the effective dielectric properties of antireflective coatings on the front glass or for multiple component AR coatings on crystalline silicon cells $[14,15]$. Our simulation does not include increased scattering caused by such a rough $\mathrm{TCO} /$ absorber interface. A more accurate treatment of rough interfaces should also involve the analysis of haze values (ratio of total integrated transmitted light to specular transmitted light). This analysis is outside the scope of this paper.

Within this work, we will not only describe the potential gain that could result from using hypothetical ideal materials, but also potential gains due to ternary and quaternary oxide materials currently available. The hypothetical materials will be referred to as ideal TA-TCO and ideal GT-TCO for the TCO/absorber and Glass/TCO interface respectively. The TCO materials currently available will be referred to by the actual chemical composition (e.g. SnO:(F, S)).

\subsection{Potential efficiency gain}

Typical TCO layer thickness $\left(d_{\mathrm{TCO}}\right)$ in current thin film solar cells are in the order of $500-2000 \mathrm{~nm}$, depending on the choice of TCO. The thickness of additional matching layers at the TCO/absorber interface $\left(d_{\mathrm{TA}}\right)$ and glass/TCO interface $\left(d_{\mathrm{GT}}\right)$ will be small in comparison to that. The glass/TCO interface layer is not a part of the electrically active layer of the cell and an efficiency increase is correlated to an increase in transmission.

For the $\mathrm{TCO} /$ absorber interface layer, a potential interface resistance or higher serial resistance of the additional layer can affect the electrical losses of the cell. A direct calculation of efficiency gains is hence more problematic. However materials to be used on both faces of the TCO layer are likely derived from ternary or quaternary modifications of existing TCO materials. In our assumption ideal materials have the same electrical properties of the original TCO and thus the total TCO thickness $\left(d_{\mathrm{TCO}}+d_{\mathrm{TA}}\right)$ and its resistivity are assumed to remain constant in the modified stack. For real materials tested here, the changes in resistivity for the matching material have been considered by reducing the TCO thickness only so much as to obtain the same sheet resistance of the combined TCO layer $\left(d_{\mathrm{TCO}}+d_{\mathrm{TA}}\right)$. In this case the total thickness of the combined TCO layer will increase with the addition of the anti reflective layer.

In order to evaluate the gain in efficiency it is not sufficient to simply use the total increase in transmission, as light with different wavelength contributes differently to the electrical output of the cell. Instead we use a weighted integral, where an increase in the transmission in regions with the highest quantum efficiency of the absorber and maxima in the spectral irradiance of the incoming light are properly accounted for. We also assume that other parameters, such as $V_{o c}$ and serial resistance, which influence the solar cell efficiency $\eta_{\text {orig }}$ remain unchanged. Essentially this concept is equivalent with the commonly used assumption, that an antireflective coating primarily increases the short current density $J_{s c}[14,16,17]$. Although the open circuit voltage for an ideal diode does increase with an increase in $J_{s c}\left(V_{o c} \sim \ln \left(J_{s c} / J_{0}-1\right)\right)$, the effect is typically small for an AR coatings in thin film solar cells, as the maximum gain in $J_{s c}$ is limited to the original reflective losses of $15-25 \%$. The consequent gain in $V_{o c}$ is hence even smaller and will be neglected in our discussion. This is substantiated by the experimental evidence for numerous AR coatings studied on crystalline silicon solar cells as well as AR coatings for the air/glass interface, where increases in $J_{s c}$ of up to $25 \%$ have been observed while at the same time $V_{o c}$ only increases by $2 \%$. [14, 18] Under these assumptions the increased efficiency of the optimised cell $\eta_{\mathrm{opt}}$ can then be directly calculated from the increase in transmission.

$$
\frac{\eta_{\mathrm{opt}}}{\eta_{\mathrm{orig}}}=\frac{\int_{E_{g}}^{\infty} \overline{\mathrm{IQE}}(\omega) \bar{F}(\omega) U(\omega) T_{\mathrm{opt}}(\omega) \mathrm{d} \omega}{\int_{E_{g}}^{\infty} \overline{\operatorname{IQE}}(\omega) \bar{F}(\omega) U(\omega) T_{\mathrm{orig}}(\omega) \mathrm{d} \omega}
$$

Where:

$$
\overline{\mathrm{IQE}}=\operatorname{IQE}(\omega) / \int \operatorname{IQE}(\omega) \mathrm{d} \omega
$$

is the area normalised internal quantum efficiency of the absorber and

$$
\bar{F}=F(\omega) / \int F(\omega) \mathrm{d} E
$$

is the area normalised spectral irradiance of the sun under AM 1.5 conditions [19].

One significant difference from the description in terms of a $J_{s c}$ increase is the introduction of the utilisation factor $U(\omega)$ :

$$
U=\left\{\begin{array}{rr}
1-\left(\omega-e V_{o c}\right) / \omega & \text { for }\left(\omega \geq E_{g}\right) \\
0 & \text { for }\left(\omega<E_{g}\right)
\end{array}\right.
$$


and the use of the spectral irradiance $F$ instead of photon flux $\Phi$. From a discussion on $J_{s c}$ only it would not matter if an electron was generated by a UV photon $\left(\omega \gg E_{g}\right)$ or by a photon with an energy close to the band gap $\left(\omega \sim E_{g}\right)$ - increasing any of the two would increase $J_{s c}$. From a conversion efficiency point of view it is more beneficial to increase the number of electrons generated by photons with lower energies, as these will show lower thermalisation losses. We estimate the thermal losses for each photon by assuming that only an energy equivalent of the open source voltage $e V_{o c}$ can be used for each electron generated by an incident photon with energy $E>E_{g}$. In this way we account for the overall spectral dependence of the electrical gain under illumination. This thermalisation loss is normally treated only in full device simulations. By using the above analytic expression we can include this effect already in simple optical models of a solar cell. The quantity $\overline{\mathrm{IQE}} \bar{F} U$ represents a weighting function and it will be shown as reference in calculated transmission curves in sections 3 and 4 . Values for the internal quantum efficiency and band gap $E_{g}$ of an a-Si:H absorber have been taken from [20], a typical open source voltage for a-Si:H cells of $V_{o c}=0.9 \mathrm{~V}$ was used in our calculations in line with values found in real devices. This weighting function is essentially a simplified energetic balance for a given absorber material, as it includes incoming power $(F)$, absorbable power (IQE) and the fraction of the absorbed power which can be used per photon by the device $(U)$.

Under these assumptions the potential gain in efficiency will be $\Delta \eta=\frac{\left(\eta_{\text {opt }}-\eta_{\text {orig }}\right)}{\eta_{\text {orig }}}$ for a given solar cell geometry with $T_{\text {orig }}$ being the transmission into the absorber layer of the original cell (see Fig. 1a), $T_{\text {opt }}$ the same for the modified cell layout (Fig. 1b,c). Using the light transmitted into the absorber as a reference is the most accurate way to asses the potential gain. However for an estimation of the effect of rough interfaces we can only apply a simulation based on reductions in the total stack reflectance $R_{\text {orig }}$ as will be discussed later. Efficiency improvements based on this will be referred to as $\Delta \eta_{R}$.

In order to optimise the thickness of the anti-reflective interface layer, a least square fit of the transmission of the stacks $T_{\text {opt }}(\omega)$ to the ideal $T=1$ was used. By weighting each point with the function $\overline{\mathrm{IQE}} \bar{F} U$, the thickness and other properties of any anti-reflective coating positioned between each layers are optimised to obtain the highest gain in efficiency as derived by equation (1). The advantage of this approach is that AR layers can be designed and assessed without the need for computational expensive device simulations.

\subsection{Materials and Experimental setup}

In order to demonstrate the effect of the proposed anti reflective layer at the glass/TCO absorber interface, test samples of glass $/ \mathrm{SnO}_{2}: \mathrm{F}$, glass/ZnO: $\mathrm{Al}$ and derived ternary oxides have been grown in house by means of spray pyrolysis. This technique allows for rapid testing of potential ternary and quaternary TCO modifications, as a wide range of materials can be synthesised, only with a change in solution [21]. At the same time it can be upscaled for industrial type deposition [22].
Best films of transparent oxides have been produced by employing organic solvents (here methanol) and organic salts [21, 23-25]. Zinc acetate dihydrate $\left(\mathrm{Zn}\left(\mathrm{CH}_{3} \mathrm{CO}_{2}\right)_{2} \cdot 2 \mathrm{H}_{2} \mathrm{O}\right)$, dibutyltin diacetate $\left(\left(\mathrm{CH}_{3} \mathrm{CH}_{2} \mathrm{CH}_{2} \mathrm{CH}_{2}\right)_{2} \mathrm{Sn}\left(\mathrm{CH}_{3} \mathrm{CO}_{2}\right)_{2}\right.$, DBTDA), aluminum acetyl acetonate $\left(\mathrm{Al}\left(\mathrm{C}_{5} \mathrm{H}_{7} \mathrm{O}_{2}\right)_{3}, \mathrm{AAA}\right)$, and magnesium acetate tetrahydrate $\left(\left(\mathrm{CH}_{3} \mathrm{CO}_{2}\right)_{2} \mathrm{Mg} \cdot 4 \mathrm{H}_{2} \mathrm{O}\right)$ were used as $\mathrm{Zn}, \mathrm{Sn}, \mathrm{Al}$, and $\mathrm{Mg}$ source respectively. Anionic dopants have been introduced with ammonium flouride $\left(\mathrm{NH}_{4} \mathrm{~F}\right)$ and thiourea $\left(\left(\mathrm{NH}_{2}\right)_{2} \mathrm{CS}\right)$ as $\mathrm{F}$ and $\mathrm{S}$ source.

The spray pyrolysis setup was developed in house and details are given elsewhere [23]. The molarity of the solution varies depending on the type of materials. For $\mathrm{ZnO}$ based materials a $0.1 \mathrm{M}$ solution of the $\mathrm{Zn}$ precursor in methanol has been used. The optimum growth temperature for plain $\mathrm{ZnO}$ was found to be $350^{\circ} \mathrm{C}$, however to improve incorporation of the co-dopants, other temperatures where required for ternary materials (e.g. $480^{\circ} \mathrm{C}$ for $\mathrm{ZnAlO}$ with high aluminium content $\left.(\mathrm{Al} / \mathrm{Zn}>0.3)\right)$. We will label such ternary samples with the nominal atomic concentration of the additional element with respect to $\mathrm{Zn}$ in the solution. The actual incorporation of the replaced anion or cation in the material could be different.

$\mathrm{SnO}_{2}$ based materials have been grown in a $0.1 \mathrm{M}$ solution of DBTDA in methanol at an optimum temperature of $450^{\circ} \mathrm{C}$. Again the optimum growth temperature can vary for the ternary compounds and the composition is given in nominal atomic concentration in the precursor solution with respect to $\mathrm{Sn}$.

The dielectric function of the TCO and modified materials has been determined by ellipsometric measurements, using a Sopra GESP 5 variable angle spectroscopic ellipsometer at three different angles of incidence close to the pseudo Brewster angle $\left(55,60,65^{\circ}\right)$. The ellipsometric data (see example in Fig. 2a) have been fitted to a model dielectric function used for bulk critical points [26]. The modelled dielectric function uses a single $3 \mathrm{D}$ bulk critical point $\left(\mathrm{SnO}_{2}\right.$ derived material) and in case of the $\mathrm{ZnO}$ derived materials an additional 1D bulk critical point to account for the strong excitonic peak present in these samples.

Figure 2 shows two typical measurements and best fit model dielectric function for a $\mathrm{SnO}_{2}$ and $\mathrm{ZnO}$ sample. By using this analysis we can parametrise the optical response of the TCO with a set of 4 values, namely the constant dielectric background $\epsilon_{\infty}$, the oscillator strength $A$, the broadening $\Gamma$ and the band gap $E_{g}$. Such a parametrisation makes a systematic analysis of modified TCO materials easier than using the raw data. For a detailed description of the physical meaning of these parameters we refer to [26]

\section{Optimised TCO/absorber interface layer}

Antireflective coatings in photovoltaic solar cells are currently only employed at the air/glass interface using $\mathrm{SiO}_{2}$ or $\mathrm{TiO}_{2}$ nanospheres. The packing density of these spheres determines the effective dielectric function of such a layer and hence can be tuned by sphere size and preparation conditions. With air as filling material between the nanospheres and a suitable sphere density, the dielectric constant of such a layer can fulfil the ideal antireflective material requirement of $n_{A R}=\sqrt{n_{\text {glass }}}$ 


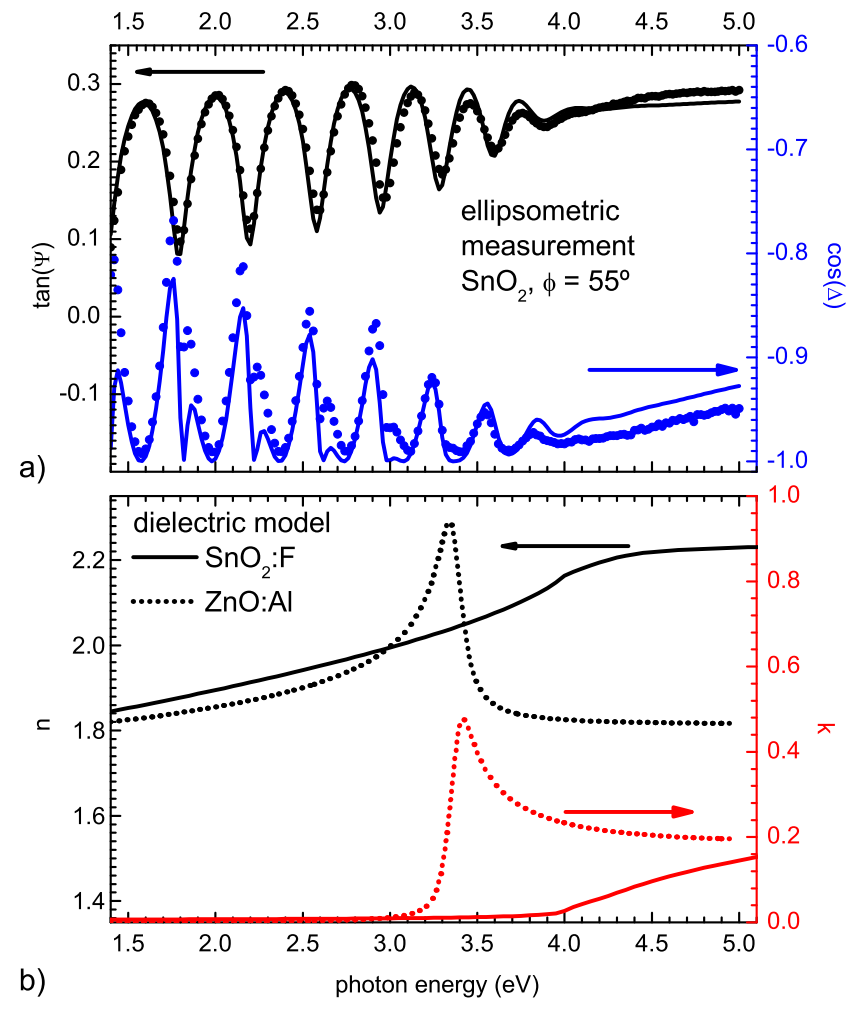

Figure 2: Representative result of the ellipsometric measurements. a) shows the ellipsometric data $\cos \Delta$ and $\tan \Psi$ for a $\mathrm{SnO}_{2}$ sample measured at $55^{\circ}$. b) shows the extracted model dielectric function for two samples derived from a simultaneous fit of measurements at 55,60 and $65^{\circ}$, using a mathematical model for bulk critical points [26]. $\mathrm{SnO}_{2}$ based samples are best fit using a 3D bulk critical point, while $\mathrm{ZnO}$ based samples are best fit with a combination of a $3 \mathrm{D}$ and $1 \mathrm{D}$ bulk critical point to account for the strong excitonic absorption at the band gap.

$[11,27]$. By employing more than a single AR layer at the top interface, the total gain can be further enhanced [27], though the net gain due to each additional AR layer decreases with the number of AR layers present and hence it is not economically viable for low cost cells.

The light loss at each internal interface is comparable to that at the front surface, but to our knowledge modern thin film cells do not widely employ purposely made AR layers to minimise these losses. Ideally, a matching layer at the TCO/absorber interface should have have a refractive index $n_{T A}=\sqrt{n_{A} n_{T}}$. The refractive index of the TCO $\left(n_{T}\right)$ is typically featureless in the spectral region of interest for PV (see Fig. 3a). The absorber layer in contrast, does show a strong dispersion. Hence it is unlikely that real materials can be perfectly matched to the line-shape of the dielectric function of the absorber. Thus minimizing the reflectance over the whole spectral range is not possible. However, by employing the weighting function during fitting, we minimize the reflectance predominately in the energy region where the absorber is most efficient. In this way the thickness and dielectric parameters are chosen in a way that the maximum of the broad Fabry-Perot oscillation due to the thin additional layer coincides with the maximum in the quantum efficiency of the cell. A significant increase in transmission can
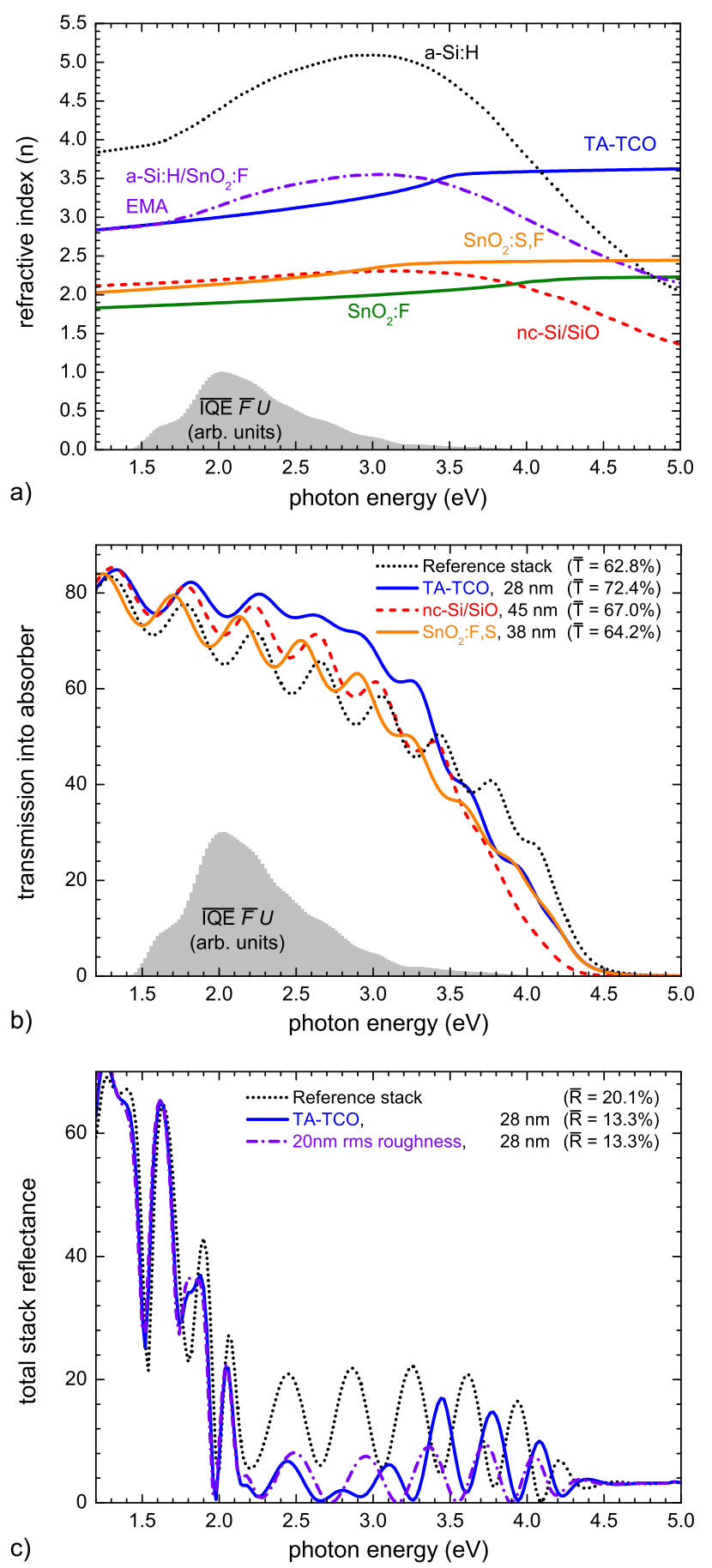

Figure 3: a) shows the refractive indices for the TCO $\left(\mathrm{SnO}_{2}: \mathrm{F}\right.$, FTO, measured), ideal AR-TCO layer (TA-TCO, simulated), a heavily sulphur doped FTO $\left(\mathrm{SnO}_{2}:(\mathrm{F}, \mathrm{S})\right.$, measured), a nanocrystalline silicon - silicon monoxide mixed layer (nc-Si/SiO: Bruggeman EMA, $85 \%$ nc-Si in $\mathrm{SiO}$, tabulated values from [28] and [29]), absorber (a-Si:H: amorphous silicon, taken from [30]), and a rough interface layer (a-Si:H/SnO 2 :F EMA: Bruggeman EMA 50\% a-Si:H in FTO). b) shows the calculated transmission into the absorber for various interface matching materials in comparison to the reference stack without them. For each material the optimum thickness and average transmission in the range of 1.5 to $3.5 \mathrm{eV}$ is given. The weighting function $\overline{\mathrm{IQE}} \bar{F} U$ as described in section 2.2 is shown both in a) and b) to illustrate the important spectral range. c) shows the total stack reflectance for the reference stack, a stack including the simulated ideal TA-TCO, and one with a rough interface layer (Bruggeman EMA 50\% a-Si:Hin FTO) 
be achieved even with a dielectric material having a featureless spectral shape, similar to that of the TCO layer. Figure 3 shows the effect of an optimised $\mathrm{TCO} /$ absorber interlayer on the transmission into the absorber for a hypothetical ideal TA-TCO and some real materials. Using (1) the potential efficiency gain of such a layer can be calculated. For the ideal TA-TCO an increase $\Delta \eta$ of $14.5 \%$ can be achieved, while the overall change in transmission is even higher, at $15 \%$.

The question remains, how such a material can be fabricated. In the case of the glass front of solar modules, an almost ideal dielectric $\mathrm{AR}$ coating can be composed of $\mathrm{SiO}_{2}$ nanospheres as discussed above. This approach can not directly be applied to an intermediate layer located at the TCO/absorber interface. Even though nanospheres of the TCO material can be synthesised, it is questionable whether embedding them in the absorber material as required for an AR material is possible and compatible with current growth techniques and cell layouts.

However we suggest that an alternative approach can be taken based on novel ternary and quaternary oxides. By addition of heavier elements into the TCO layer, the dielectric function of a given TCO can be modified. The dielectric function of such a material is most likely inferior to that of the ideal TATCO shown in Fig. 3a. However if one incorporates our current best candidate, heavily sulphur doped FTO $\left(\mathrm{SnO}_{2}:(\mathrm{F}, \mathrm{S})\right)$, into the reference structure as shown in Fig. 1b,c, using a thickness of $37 \mathrm{~nm}$, cell efficiencies are expected to increase by as much as $\Delta \eta=4.5 \%$. We hereby assume that any disimprovement in electrical properties of the cell is negligible. In fact, based on the resistivity values for the modified TCO of $0.15 \Omega \mathrm{cm}$, a change in sheet resistance of the overall TCO stack due to the thin high resistive AR layer would only be $0.3 \%$. Other parameters affecting the cell performance, such as band offsets and changed serial resistance can not be estimated at present.

An alternative approach to modifying the TCO material to match it to the properties of the absorber material is to modify the absorber material itself. Recently it was reported that including a thin $\mathrm{SiO}_{\mathrm{x}}$ layer grown at highly oxygen-deficient conditions can improve cell performance due to the effect described here [6]. The $\mathrm{SiO}_{\mathrm{x}}$ synthesised in these conditions is a meta-material consisting of n-type $\mathrm{SiO}_{\mathrm{x}}$ and $\mathrm{Si}$ nanoclusters. The material was embedded at the $\mathrm{ZnO}: \mathrm{Al} / \mathrm{a}-\mathrm{Si}: \mathrm{H}$ interface in thin film a-Si:H/ $\mu \mathrm{c}-\mathrm{Si}$ tandem cells. Such a material is compatible with the production process, as it can be grown in a similar PECVD system as used for the absorber. However this meta-material can not be used with other thin film cells and furthermore due to the additional absorption of the nanocrystalline silicon within the matrix it is not a good AR material. For comparison we included the results of a simulation based on such a layer in Fig. $3 \mathrm{~b}$ and Table 1 (nc-Si/SiO). A Bruggeman effective medium mixture of silicon monoxide $(\mathrm{SiO})$ and nanocrystalline-silicon (nc-Si) was used to describe the dielectric properties of this particular matching material. The thickness and EMA fill factor have been fitted for a maximised transmission into the absorber layer in the same way as described above for our hypothetical modified TCO. Based on the database dielectric function for nc-Si $(4 \mathrm{~nm})$ and $\mathrm{SiO}$ taken from [28] and [29], a gain of $\Delta \eta=7.8 \%$ can be achieved if the match- ing material has a thickness of $45 \mathrm{~nm}$ and consists of $85 \% \mathrm{nc}-\mathrm{Si}$ and $15 \% \mathrm{SiO}$. The absolute value does strongly depend on the dielectric functions used in the calculation. Larger quantities of $\mathrm{SiO}_{2}$ in the meta-material would lower its refractive index too much, while larger quantities of $\mathrm{SiO}$ or larger silicon crystals would increase its absorption. Measurements of the refractive index of $\mathrm{Si}$ rich $\mathrm{SiO}_{x}$ grown by PECVD have shown this behaviour clearly [31]. The highest $\mathrm{Si}$ content investigated was $20 \%$, which only resulted in a refractive index of $\sim 2.1$, too low for an effective TCO/a-Si:H antireflective coating material. This shows that such an $\mathrm{SiO}_{\mathrm{x}}$ meta-material is not an ideal choice due to limitations in the achievable optical properties and explains the relatively small measured increase of $\Delta \eta=2.3 \%$ as reported in [6].

A rough TCO/absorber interface frequently employed in thin film solar cells does have an effect similar to the one described here $[32,33]$. Any rough interface with feature sizes smaller than the wavelength of the incident light acts as an effective medium interlayer, or graded interface. In this case the reflectance of the interface is reduced. Part of the light is scattered though, which leads to an effective reduction in the specular transmission. In the case of the $\mathrm{TCO} /$ absorber, interface scattering is not problematic as any forward scattered light still reaches the absorber. The change can even be desirable, as the optical path length within the absorber increases for the scattered light $[7,34]$. It is often reported, that the increase in efficiency for a rough $\mathrm{TCO} / \mathrm{absorber}$ interface is caused by multiple reflections similar to the case of large scale textured front surfaces or crystalline silicon textures [35]. For low haze TCOs the structure size is much smaller than the wavelength, hence geometrical optics can not be applied $[11,36]$. We suggest instead that the increase in efficiency is caused by the introduction of an effective medium layer at this interface in the same way as nano structured glass textures can reduce reflection from the front glass [15]. This idea is also supported by earlier numerical simulations $[37,38]$. A rough interface will be treated as an effective medium layer using the Bruggeman approximation with fill factor 0.5 . The thickness of the interfacial layer is related to the rms roughness: $d_{A R}=\sqrt{2} \mathrm{rms}$, corresponding to a surface profile approximated by a $|\sin (x)|$ line shape. Unlike the previous case, for a rough interface we have to base our calculations on the decrease in overall reflectance, instead of the increase in transmission to the absorber. This is required as an effective medium model results in an increased absorption of the light in the interface layer, which leads to a lower effective transmission into the simulated absorber. In a real cell, the light absorbed in the interface region is still collected, as absorbing areas of the mixed medium are electrically connected to the absorber and hence still contribute to the performance of the cell. However, reliance on overall reflectance in the calculations introduces some systematic differences compared to the use of transmission into the absorber. The latter assumes that the absorber utilises all transmitted light, while the former does include the fact that for a poor absorber, such as a-Si:H, a significant amount of light is reflected from the back metallisation and leaves the absorber again through the front window. The extent of that additional loss depends on absorber thickness and 
can be minimised by increasing it. The gains calculated taking into consideration the overall Reflectance $\left(\Delta \eta_{R}\right)$ are more realistic than those calculated by the transmission $\left(\Delta \eta_{T}\right)$ for a poor absorber, but in general the former method tends to underestimate the full potential of anti-reflecting layers. For example the simulation for the ideal TA-TCO shows an expected gain of $\Delta \eta=14.5 \%$ based on transmission into the absorber, but only $\Delta \eta_{R}=8.6 \%$ based on the reflectance of the whole stack. The difference is due to a significant amount of light not being absorbed by a $300 \mathrm{~nm}$ thick a-Si:H absorber used in our calculations. For a thick absorber or one with higher absorption coefficient (e.g. $\mu c-\mathrm{Si}, \mathrm{CuInS}_{2}$ or other chalcopyrites) the value of $\Delta \eta$ and $\Delta \eta_{R}$ will be identical.

Using $\Delta \eta_{R}$ we predict an increase in cell efficiency of $8.3 \%$ and $7.7 \%$ for a rms roughness of 20 and $40 \mathrm{~nm}$. It is noteworthy, that this is already close to the potential gain of an ideal TA-TCO $(8.6 \%)$ using the same reflectance based model. As seen in Fig. 3 a there is little difference in the refractive index of the ideal TA-TCO and the effective medium dielectric function used to simulate the rough layer in the interesting energy region of $1.5-3 \mathrm{eV}$.

For low haze TCO (roughness of 20-40 nm [39]) our calculation is meaningful as a small range roughness does not significantly change the light path through scattering. Nevertheless small scale roughness has been reported to equally increase cell efficiencies as high haze TCOs [33, 40]. In simple words, the comparisons between the results reported in literature with those obtained from our model calculation, led us to conclude that for low haze values, the grading effect is the main cause for the improvement in the cell efficiency. For a large scale roughness $(\mathrm{rms}>60 \mathrm{~nm}$ ) our model can not be applied though. First a better simulation would require a graded index layer [15], a model more complex than the Bruggeman approximation. Furthermore the scattering effect is important for an interface with large roughness. In this case the light path is significantly altered by scattering, thus enhancing the chance for a photon to be absorbed. For these reasons our model underestimates the gain for a large roughness.

\section{Optimised glass/TCO interface layer}

Using a similar approach of introducing additional elements in the TCO lattice, the refractive index of the TCO material can be lowered to create an index-matched material for the glass/TCO interface (GT). As this particular layer is not located in the electrically active region of the solar cell, a much wider range of materials can be discussed as non conductive oxides can be equally used. The key problem is finding an ideally index matched material with a very low absorbance.

One candidate we have chosen, is the spinel $\mathrm{MgAl}_{2} \mathrm{O}_{4}$ as its reported refractive index at $600 \mathrm{~nm}$ of $n \sim 1.7$ is well suited for the interface between glass $(n \sim 1.5)$ and the TCO materials $(n \sim 1.95)$.

Secondly, we have prepared an amorphous test material based on a modified $\mathrm{ZnO}: \mathrm{Al}$ (AZO) We observe an increase in the band gap and lower refractive index with increasing aluminium content (see Fig. 4). For small quantities of $\mathrm{Al}(1-4 \%)$

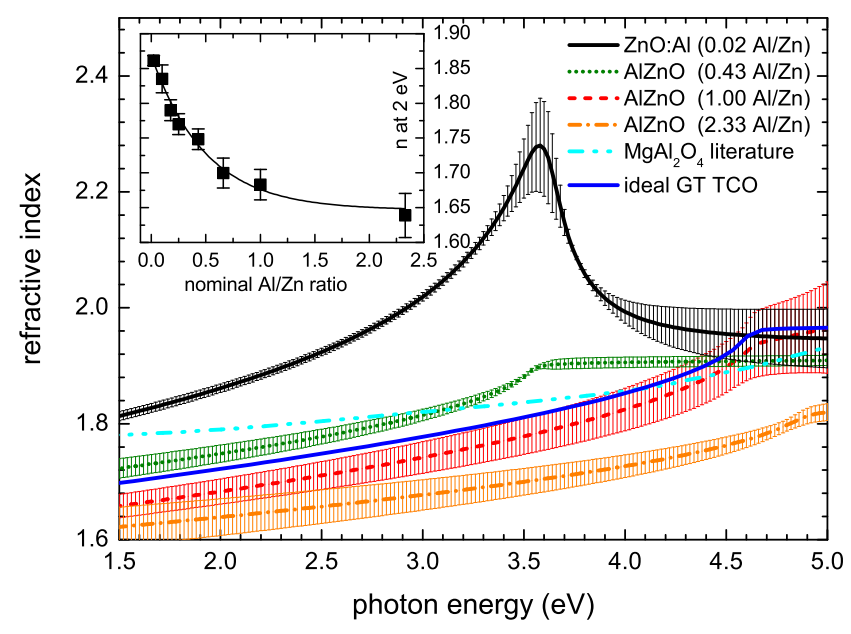

Figure 4: Measured optical constants of $\mathrm{Al}_{\mathrm{y}} \mathrm{Zn}_{(1-\mathrm{y})} \mathrm{O}_{\mathrm{x}}$ layers with varying nominal $\mathrm{Al} / \mathrm{Zn}$ ratio in the precursor solution. The decrease in the refractive index with growing $\mathrm{Al}$ content is obvious. In addition the literature values [41] for a thin film $\mathrm{MgAl}_{2} \mathrm{O}_{4}$ spinel and the hypothetical ideal GT-TCO are also shown. The inset shows the reduction in $n$ at $2.0 \mathrm{eV}(620 \mathrm{~nm})$ with the increase in $\mathrm{Al} / \mathrm{Zn}$ ratio. The nominal $\mathrm{Al} / \mathrm{Zn}$ ratio is defined by the atomic concentrations of the aluminium and zinc precursors in the sprayed solution.

the material is the well known conductive $\mathrm{ZnO}: \mathrm{Al}$, with the typical strong excitonic absorption peak close to the band gap. The microscopic structure of the films grown with higher aluminium concentrations $(>10 \%)$ is currently not known, as no crystalline phases are found. We therefore assume the material is amorphous, with local bond geometries changing from $\mathrm{ZnO}$ to those of $\mathrm{Al}_{2} \mathrm{O}_{3}$ with increasing $\mathrm{Al}$ content. The absolute atomic ratio of $\mathrm{Al} / \mathrm{Zn}$ in the films is also not known at present time, as the actual incorporation is a complex function of known precursor concentration and unknown parameter such as precursor decomposition rates and sticking coefficients. The optical properties have been measured on several films with thickness between 300 and $500 \mathrm{~nm}$. Variations between individual films and systematic errors in the determination of $n$ from the ellipsometric measurements are indicated in Fig. 4 by error bands.

Figure 5 shows the calculated increase in transmission for stacks consisting of glass $/ \mathrm{SnO}_{2}: \mathrm{F} / \mathrm{a}-\mathrm{Si}: \mathrm{H}$ as a reference and such using $\mathrm{AlZnO}_{\mathrm{x}}, \mathrm{MgAl}_{2} \mathrm{O}_{4}$, and the ideal GT-TCO at the glass $/ \mathrm{SnO}_{2}: \mathrm{F}$ interfae. Measured dielectric functions have been used for $\mathrm{SnO}_{2}: \mathrm{F}$ and $\mathrm{AlZnO}_{\mathrm{x}}$, while database dielectric functions have been used for $\mathrm{MgAl}_{2} \mathrm{O}_{4}$ and a-Si:H [30, 41].

The calculation for the ideal GT-TCO is again based on a model dielectric function used for $\mathrm{SnO}_{2}: \mathrm{F}$ with a changed $\epsilon_{\infty}$, $A, \Gamma, E_{g}$, to maximise the transmission into the absorber. The potential increase in transmission into the absorber layer for $\mathrm{AlZnO}_{\mathrm{x}}$ with nominal $\mathrm{Al} / \mathrm{Zn}$ ratio of 0.67 (see Fig. 5 inset) is comparable to the ideal GT-TCO, though the film is not conductive. This has implications for the potential cost reduction details of which are discussed in the following section. In the ideal case an increase in transmission into the absorber of $2.3 \%$ is possible. If the weighting function $\overline{\mathrm{IQE}} \bar{F} U$ is considered, this translates into an efficiency gain of $1.9 \%$. By using the materials $\mathrm{MgAl}_{2} \mathrm{O}_{4}, \mathrm{AlZnO}_{\mathrm{x}}(\mathrm{Al} / \mathrm{Zn}=1)$, and $\mathrm{Al}_{0.4} \mathrm{Zn}_{0.6} \mathrm{O}_{\mathrm{x}}$ 


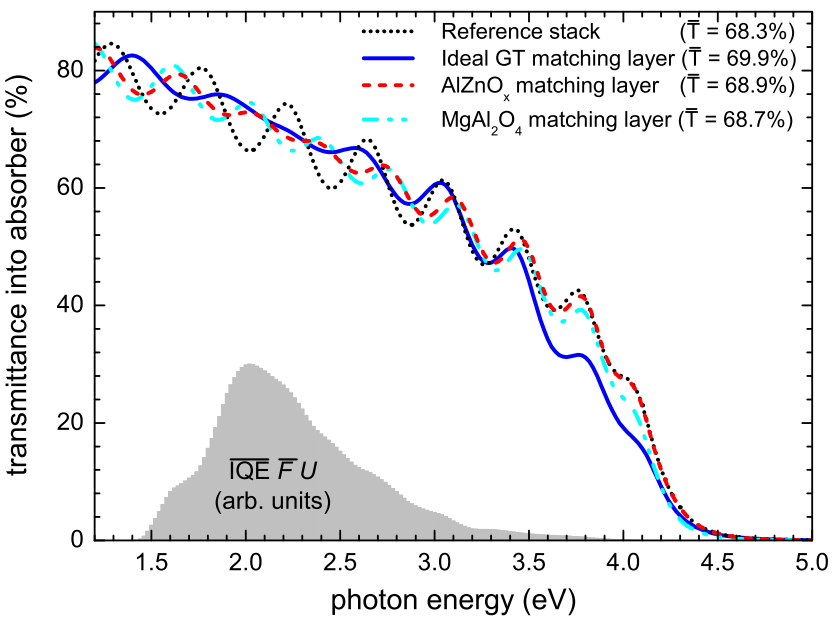

Figure 5: Transmission into the absorber for the reference stack, the ideal matching material for the glass/TCO interface (GT-TCO, $\left.d_{A R}=81 \mathrm{~nm}\right)$, our best matched material available $\mathrm{AlZnO}_{\mathrm{x}} d_{A R}=81 \mathrm{~nm}$, and $\mathrm{MgAl}_{2} \mathrm{O}_{4} d_{A R}=78 \mathrm{~nm}$. The ideal thickness was determined by a full optimisation of the stack with respect to the maximum efficiency gain as given by (1) using the weighting function indicated by the grey area. The number in brackets is the average transmission between 1.4 and $3 \mathrm{eV}$

$(\mathrm{Al} / \mathrm{Zn}=0.67)$ an efficiency gain of $0.6,1.1$, and $1.7 \%$ is predicted. For these real materials the expected efficiency gain is reduced as the refractive index deviates further from the ideal matched case (see Fig. 4). Although the introduction of glass/TCO matching layers does increase the efficiency, the gain is less significant, as the reflective losses at that interface are smaller compared to the losses at the air/glass and TCO/absorber interfaces. The measure can still be justified if the additional cost due to the insertion of the layer is small in comparison to the cost benefit arising from the improved efficiency as discussed in the next section.

\section{Cost structure}

We have shown that the employment of internal antireflective layers can improve the efficiency of thin film solar cells. Usually the introduction of new layers complicates the production and increases material and equipment costs. We have demonstrated that the candidate materials for additional matching layers can be grown using the same technique and precursor materials as the TCO layers themselves. We can therefore expect no significant differences in equipment costs between chambers used for TCO growth and those used for AR layers. The increased material costs are hence directly related to the thickness of the grown layer. In the best case scenario of the ideal matching materials we also assume its resistivity is similar to that of conventional TCOs. In that case the original TCO thickness $d_{T C O}$ can be reduced by the thickness $d_{A R}$ of the matching layer, leaving the total thickness and costs constant. Hence the ideal matching materials are always economically beneficial.

For the non ideal material from the pool of known matching materials, an increase in the total thickness of the TCO structure is required, as the TCO layer thickness remains unchanged or

\begin{tabular}{|c|c|c|c|c|}
\hline Material & $n_{\mathrm{AR}}$ & $d_{\mathrm{AR}}(\mathrm{nm})$ & $\Delta d(\%)$ & $\Delta \eta(\%)$ \\
\hline \multicolumn{4}{|c|}{ glass/TCO interface, transmission based } & $(\Delta \eta)$ \\
\hline ideal GT-TCO & 1.72 & 81 & - & 1.9 \\
\hline $\mathrm{MgAl}_{2} \mathrm{O}_{4}$ & 1.8 & 78 & 12 & 0.6 \\
\hline $\mathrm{AlZnO}_{\mathrm{x}}(\mathrm{Al} / \mathrm{Zn}: 1)$ & 1.68 & 82 & 12.5 & 1.1 \\
\hline $\mathrm{Al}_{0.4} \mathrm{Zn}_{0.6} \mathrm{O}_{\mathrm{x}}(\mathrm{Al} / \mathrm{Zn}: 0.67)$ & 1.70 & 81 & 12.5 & 1.7 \\
\hline \multicolumn{4}{|c|}{ TCO/a-Si interface, transmission based } & $(\Delta \eta)$ \\
\hline ideal TA-TCO & 2.8 & 28 & - & 14.5 \\
\hline $\mathrm{SnO}_{2}: \mathrm{S}, \mathrm{F}$ & 2.1 & 37 & 6 & 4.5 \\
\hline nc-Si/SiO & 2.2 & 45 & 7 & 7.8 \\
\hline \multicolumn{4}{|c|}{ TCO/a-Si interface, reflectance based } & $\left(\Delta \eta_{R}\right)$ \\
\hline ideal TA-TCO & 2.8 & 28 & - & 8.6 \\
\hline rough TCO $(20 \mathrm{~nm})$ & 3.4 & 28 & - & 8.3 \\
\hline rough TCO $(40 \mathrm{~nm})$ & 3.4 & 57 & - & 7.7 \\
\hline
\end{tabular}

Table 1: Front contact relative thickness increase $\Delta d$ (for an original TCO thickness $d$ of $650 \mathrm{~nm}$ ) vs. gain estimation $\Delta \eta$ for several glass / TCO and TCO / absorber antireflective layers. Also listed are the measured or estimated refractive index of the AR coating material $\left(n_{\mathrm{AR}}\right)$ at $525 \mathrm{~nm}$, the calculated optimum thickness $d_{\mathrm{AR}}$ for the particular material. The calculated efficiency gain $\Delta \eta$ was evaluated using Eq. (1). For comparison with rough interfaces $\Delta \eta_{R}$ calculations, based on a minimisation of the overall reflection resulting in systematically lower gain estimations are given as well (see text, section 3 ).

is only slightly reduced to ensure the same sheet resistance of the front contact as in the reference stack.

In table 1 we compare this increase in thickness $\Delta d$ with the efficiency gain $\Delta \eta . \Delta d$ is based on an assumed original TCO thickness of $650 \mathrm{~nm}$ used in all our calculations. According to the $\Delta d$ and $\Delta \eta$ values, the addition of an anti-reflecting layer will be more cost-efficient for thicker TCO absorber layers, as $\Delta d$ decreases. Nevertheless $\Delta d$ approximates only the increasing costs of the overall TCO layer. As only a fraction of the total solar cell cost originates from the TCO deposition, the efficiency gain $\Delta \eta$ needs to be compared to the change in total system costs. Based on the calculated changes in total stack thickness all the currently available candidate materials for the TCO/absorber interface would improve $\operatorname{cost} / W_{p}$ if the TCO layer contributes less than $75 \%$ of the total cell costs, while for the best glass TCO absorber interface material found so far $\left(\mathrm{Al}_{0.4} \mathrm{Zn}_{0.6} \mathrm{O}_{\mathrm{x}}, \mathrm{Al} / \mathrm{Zn}\right.$ : 0.67$)$ the inclusion is only helpful if the TCO contributes less than $14 \%$, based again on a $650 \mathrm{~nm}$ thick TCO layer. The situation at the glass/TCO interface would improve if the matching layer itself would be conductive as in this case the will be no need to increase the thickness. Doping mechanisms for the amorphous $\mathrm{AlZnO}_{\mathrm{x}}$ with high $\mathrm{Al}$ content are currently under investigation in our lab.

\section{Conclusion}

We have analysed the potential efficiency gain of glass/TCO and TCO/absorber intermediate layers for thin film solar cells. Quaternary oxides derived from known TCO materials can show the optical properties required for such layers. With the materials $\mathrm{AlZnO}_{\mathrm{x}}$ and $\mathrm{SnO}_{2}: \mathrm{S}, \mathrm{F}$ a relative efficiency gain of $\Delta \eta=6.2 \%$ could be achieved if they were incorporated into an a-Si:H thin film solar cell. A cell with e.g $\eta_{\text {orig }}=9 \%$ would hence show an increased efficiency of $\eta_{\text {opt }}=9.6 \%$ by employing these materials. Further increases in gain could be achieved, if the 
materials with optimised optical constants as per our theoretical models became available. For such ideal matching materials we predict efficiency gains $\Delta \eta$ of up to $16 \%$, or in absolute terms an increase of $\eta_{\text {orig }}=9 \%$ to $\eta_{\text {opt }}=10.4 \%$.

We have furthermore demonstrated, that the reported gain of rough $\mathrm{TCO} /$ absorber interfaces with low haze values, can be explained by the anti-reflective properties of the rough interface acting as an effective medium with an refractive index between values of $\mathrm{SnO}_{2}: \mathrm{F}$ and a-Si:H. In the latter case we have neglected potential additional gains due to the change in the effective absorber thickness for the scattered light. In contrast, matching layers based on $\mathrm{SiO}$ and nc-Si are shown to be helpful, but by far, non-ideal matching materials.

Finally we have shown that if the cost of such ideal interface materials is comparable to those of the TCO itself, the inclusion of such layers can not only increase the total efficiency but more importantly reduce the relevant cost $/ \mathrm{W}_{p}$ of thin film solar cells. Although we only analysed a relatively simple single absorber cell structure, the concept can be applied to any other type of solar cells with glass/TCO or TCO/absorber interfaces.

\section{Acknowlegements}

The authors would like to acknowledge financial support from Enterprise Ireland under grant PC/2007/0367 and SFI 06/IN.1/I91 TIDA Feasibility 10.

[1] K. L. Chopra, P. D. Paulson, V. Dutta, Thin-film solar cells: An overview, Prog. Photovoltaics 12 (2004) 69.

[2] M. A. Green, Consolidation of thin-film photovoltaic technology: The coming decade of opportunity, Prog. Photovoltaics 14 (2006) 383.

[3] W. Shockley, H. J. Queisser, Detailed Balance Limit of Efficiency of P-N Junction Solar Cells, J. Appl. Phys. 32 (1961) 510.

[4] W. Beyer, J. Hupkes, H. Stiebig, Transparent conducting oxide films for thin film silicon photovoltaics, Thin Solid Films 516 (2007) 147.

[5] M. Kursawe, R. Anselmann, V. Hilarius, G. Pfaff, Nano-particles by wet chemical processing in commercial applications, J. Sol-Gel Sci. Technol. 33 (2005) 71.

[6] S. Klein, S. Wieder, S. Buschbaum, K. Schwanitz, T. Stolley, D. Severin, P. Obermeyer, M. Kress, E. Sommer, T. Marschner, M. Martini, S. NollBaumann, J. Haack, U. Schmidt, A. Straub, K. Ahmed, K. Schuegraf, Large area thin film solar modules with $10 \%$ efficiency for mass production, 25th European Photovoltaic Solar Energy Conference, p. 2708.

[7] J. Müller, B. Rech, J. Springer, M. Vanecek, TCO and light trapping in silicon thin film solar cells, Sol. Energy 77 (2004) 917.

[8] S. Y. Guo, L. Sahoo, G. Sosale, A. E. Delahoy, Textured, doped, ZnO thin films produced by a new process for a-Si and CIGS solar cell application, volume 6651 of SPIE 6651, p. 66510B.

[9] J. Krc, B. Lipovsek, M. Bokalic, A. Campa, T. Oyama, M. Kambe, T. Matsui, H. Sai, M. Kondo, M. Topic, Potential of thin-film silicon solar cells by using high haze TCO superstrates, Thin Solid Films 518 (2010) 3054.

[10] S. S. Hegedus, R. Kaplan, Analysis of quantum efficiency and optical enhancement in amorphous Si p-i-n solar cells, Prog. Photovoltaics 10 (2002) 257.

[11] M. Born, E. Wolf, A. B. d. Bhatia, Principles of optics : electromagnetic theory of propagation, interference and diffraction of light, Cambridge University Press, Cambridge, 7th (expanded) edition, 1999.

[12] C. C. Katsidis, D. I. Siapkas, General transfer-matrix method for optical multilayer systems with coherent, partially coherent, and incoherent interference, Applied Optics 41 (2002) 3978.

[13] D. E. Aspnes, J. B. Theeten, Investigation of Effective-Medium Models of Microscopic Surface-Roughness by Spectroscopic Ellipsometry, Phys. Rev. B 20 (1979) 3292.
[14] L. Zighed, A. Mahdjoub, Comparative Study Between Various Antireflective Coatings for Solar Cells Applications, volume 13 of IOP Conf. Ser, Mater. Sci. Eng. (UK), p. 012037.

[15] A. Gombert, W. Glaubitt, K. Rose, J. Dreibholz, B. Blasi, A. Heinzel, D. Sporn, W. Doll, V. Wittwer, Subwavelength-structured antireflective surfaces on glass, Thin Solid Films 351 (1999) 73.

[16] D. J. Aiken, High performance anti-reflection coatings for broadband multi-junction solar cells, Sol. Energ. Mat. Sol. Cells 64 (2000) 393.

[17] J. Hofstetter, C. Del Canizo, S. Ponce-Alcantara, A. Luque, Optimisation of SiNx:H anti-reflection coatings for silicon solar cells, 2007 Spanish Conference on Electron Devices, Proceedings, p. 131.

[18] T. Stapinski, B. Swatowska, Amorphous hydrogenated silicon-carbon as new antireflective coating for solar cells, J. Non-Cryst. Solids 352 (2006) 1406.

[19] ASTM, 2003. http://rredc.nrel.gov/solar/spectra/am1.5/.

[20] H. Y. Liu, L. Jiao, S. Semoushkina, C. R. Wronski, Distribution of charged defects in a:Si-H n-i Schottky barrier solar cells, J. Non-Cryst. Solids 198 (1996) 1168.

[21] P. S. Patil, Versatility of chemical spray pyrolysis technique, Mater. Chem. Phys. 59 (1999) 185.

[22] S. Aukkaravittayapun, N. Wongtida, T. Kasecwatin, S. Charojrochkul, K. Unnanon, P. Chindaudom, Large scale F-doped SnO2 coating on glass by spray pyrolysis, Thin Solid Films 496 (2006) 117.

[23] E. Arca, K. Fleischer, I. V. Shvets, Influence of the Precursors and Chemical Composition of the Solution on the Properties of $\mathrm{ZnO}$ Thin Films Grown by Spray Pyrolysis, J. Phys. Chem. C 113 (2009) 21074.

[24] X. Zhi, G. Y. Zhao, T. Zhu, Y. Li, The morphological, optical and electrical properties of $\mathrm{SnO} 2: \mathrm{F}$ thin films prepared by spray pyrolysis, Surf. Interface Anal. 40 (2008) 67.

[25] K. Omura, P. Veluchamy, M. Tsuji, T. Nishio, M. Murozono, A Pyrosol Technique to Deposit Highly Transparent, Low-Resistance SnO2:F Thin Films from Dimethyltin Dichloride, J. Electrochem. Soc. 146 (1999) 2113.

[26] P. Lautenschlager, M. Garriga, L. Vina, M. Cardona, TemperatureDependence of the Dielectric Function and Interband Critical-Points in Silicon, Phys. Rev. B 36 (1987) 4821.

[27] M. J. Minot, Single-Layer, Gradient Refractive-Index Antireflection Films Effective from 0.35 to 2.5-Mu, J. Opt. Soc. Am. A 66 (1976) 515.

[28] M. I. Alonso, I. C. Marcus, M. Garriga, A. R. Goni, J. Jedrzejewski, I. Balberg, Evidence of quantum confinement effects on interband optical transitions in Si nanocrystals, Phys. Rev. B 82 (2010).

[29] A. Szekeres, T. Nikolova, A. Paneva, I. Lisovskyy, P. E. Shepeliavyi, G. Y. Rudko, Effect of Si nanoparticles embedded in $\mathrm{SiO}_{x}$ on optical properties of the films studied by spectroscopic ellipsometry and photoluminescence spectroscopy, Optical Materials 30 (2008) 1115.

[30] A. S. Ferlauto, G. M. Ferreira, J. M. Pearce, C. R. Wronski, R. W. Collins, X. M. Deng, G. Ganguly, Analytical model for the optical functions of amorphous semiconductors from the near-infrared to ultraviolet: Applications in thin film photovoltaics, J. Appl. Phys. 92 (2002) 2424.

[31] S. Hernandez, P. Pellegrino, A. Martinez, Y. Lebour, B. Garrido, R. Spano, M. Cazzanelli, N. Daldosso, L. Pavesi, E. Jordana, J. M. Fedeli, Linear and nonlinear optical properties of $\mathrm{Si}$ nanocrystals in $\mathrm{SiO}_{2}$ deposited by plasma-enhanced chemical-vapor deposition, J. Appl. Phys. 103 (2008).

[32] A. V. Shah, H. Schade, M. Vanecek, J. Meier, E. Vallat-Sauvain, N. Wyrsch, U. Kroll, C. Droz, J. Bailat, Thin-film silicon solar cell technology, Prog. Photovoltaics 12 (2004) 113.

[33] S. Sheng, Y. K. Chae, X. Yang, J. Su, D. Wang, D. Tanner, C. Eberspacher, High performance amorphous-microcrystalline $\mathrm{Si}$ tandem solar cells and modules fabricated on next-generation TCO glass using large-area PECVD cluster systems, 24th European Photovoltaic Solar Energy Conference, p. 2850.

[34] C. Rockstuhl, S. Fahr, K. Bittkau, T. Beckers, R. Carius, F. J. Haug, T. Soderstrom, C. Ballif, F. Lederer, Comparison and optimization of randomly textured surfaces in thin-film solar cells, Optics Express 18 (2010) 1302.

[35] R. Dewan, M. Marinkovic, R. Noriega, S. Phadke, A. Salleo, D. Knipp, Light trapping in thin-film silicon solar cells with submicron surface texture, Optics Express 17 (2009) 23058.

[36] Y. A. Kravtsov, Y. I. Orlov, Limits of applicability of the method of geometric optics and related problems, Soviet Physics Uspekhi 23 (1980) 
750.

[37] J. H. van den Berg, M. Zeman, J. W. Metselaar, Optical properties of aSiGe:H solar cells on textured substrates, J. Non-Cryst. Solids 227 (1998) 1262.

[38] J. Krc, F. Smole, M. Topic, Potential of light trapping in microcrystalline silicon solar cells with textured substrates, Prog. Photovoltaics 11 (2003) 429.

[39] S. Ulrich, W. Dewald, B. Szyszka, Comparison of SnO2:F based transparent conducting oxides for us in a-Si:H/ $\mathrm{Mc}-\mathrm{Si}: \mathrm{H}$ solar cells, 24th European Photovoltaic Solar Energy Conference, p. 2814.

[40] J. Springer, B. Rech, W. Reetz, J. Muller, M. Vanecek, Light trapping and optical losses in microcrystalline silicon pin solar cells deposited on surface-textured glass/ZnO substrates, Sol. Energ. Mat. Sol. Cells 85 (2004) 1.

[41] S. M. Hosseini, Structural, electronic and optical properties of spinel $\mathrm{MgAl}_{2} \mathrm{O}_{4}$ oxide, phys. stat. sol. (b) 245 (2008) 2800. 